\title{
The association of lung cancer with pulmonary fibrosis
}

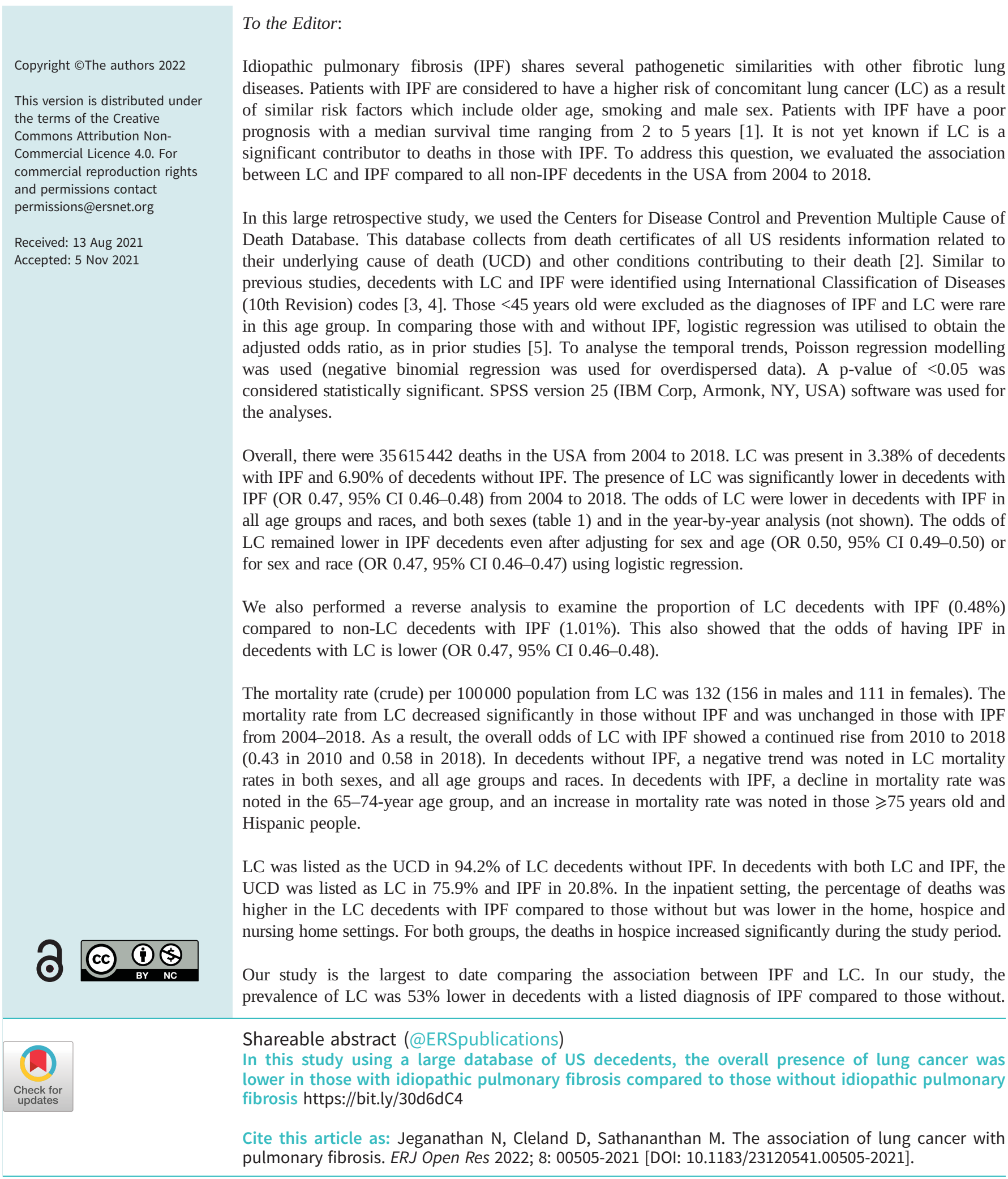


TABLE 1 Prevalence of lung cancer (LC) in decedents with and without idiopathic pulmonary fibrosis (IPF)

\begin{tabular}{|c|c|c|c|c|c|c|c|c|c|c|c|}
\hline \multirow[t]{2}{*}{ Variable } & \multicolumn{5}{|c|}{ Decedents without IPF ${ }^{\#}$} & \multicolumn{5}{|c|}{ Decedents with IPF" } & \multirow[t]{2}{*}{$\mathrm{OR}^{+}(95 \% \mathrm{Cl})$} \\
\hline & $\begin{array}{l}\text { Total } \\
\text { deaths }\end{array}$ & $\begin{array}{c}\text { LC } \\
\text { present }\end{array}$ & $\begin{array}{c}\text { Deaths with LC, } \\
\% \text { total }\end{array}$ & $\begin{array}{l}\text { LC mortality rate, } \\
\% \text { change } \\
2004-2018\end{array}$ & $\begin{array}{l}p \text {-value for } \\
\text { trend }\end{array}$ & $\begin{array}{l}\text { Total } \\
\text { deaths }\end{array}$ & $\begin{array}{c}\text { LC } \\
\text { present }\end{array}$ & $\begin{array}{c}\text { Deaths with LC, } \\
\% \text { total }\end{array}$ & $\begin{array}{l}\text { LC mortality rate, } \\
\% \text { change } \\
2004-2018\end{array}$ & $\begin{array}{l}\mathrm{p} \text {-value for } \\
\text { trend }\end{array}$ & \\
\hline \multicolumn{12}{|l|}{ Sex } \\
\hline Female & 18002346 & 1087001 & 6.04 & -23.4 & $<0.001$ & 160389 & 3985 & 2.48 & & 0.30 & $0.40(0.38-0.41)$ \\
\hline Male & 17263723 & 1346703 & 7.80 & -41.0 & $<0.001$ & 188984 & 7816 & 4.13 & & 0.32 & $0.51(0.50-0.52)$ \\
\hline \multicolumn{12}{|l|}{ Age, years } \\
\hline $45-54$ & 2677125 & 164280 & 6.14 & -48.4 & $<0.001$ & 10476 & 294 & 2.81 & & 0.26 & $0.44(0.39-0.50)$ \\
\hline $55-64$ & 4797685 & 482281 & 10.05 & -34.8 & $<0.001$ & 32043 & 1533 & 4.78 & & 0.12 & $0.45(0.43-0.47)$ \\
\hline $65-74$ & 6566472 & 767181 & 11.68 & -42.0 & $<0.001$ & 76494 & 4070 & 5.32 & -19.1 & 0.04 & $0.42(0.41-0.44)$ \\
\hline $75-84$ & 9574477 & 729948 & 7.62 & -27.4 & $<0.001$ & 126992 & 4327 & 3.40 & +22.3 & 0.001 & $0.43(0.41-0.44)$ \\
\hline$\geqslant 85$ & 11650310 & 290014 & 2.49 & -9.2 & $<0.001$ & 103368 & 1577 & 1.53 & +21.8 & $<0.001$ & $0.61(0.58-0.64)$ \\
\hline \multicolumn{12}{|l|}{ Race } \\
\hline Native-American & 186275 & 11424 & 6.13 & -12.4 & 0.02 & 2775 & 84 & 3.03 & & 0.52 & $0.48(0.38-0.59)$ \\
\hline Asian & 738695 & 49661 & 6.72 & -17.0 & $<0.001$ & 8726 & 214 & 2.45 & & 0.85 & $0.35(0.30-0.40)$ \\
\hline Black & 3851698 & 250914 & 6.51 & -32.4 & $<0.001$ & 18707 & 642 & 3.43 & & 0.34 & $0.51(0.47-0.55)$ \\
\hline White & 28498575 & 2039321 & 7.16 & -23.1 & $<0.001$ & 291706 & 10302 & 3.53 & & 0.09 & $0.47(0.47-0.48)$ \\
\hline Hispanic & 1902714 & 77398 & 4.07 & -31.2 & $<0.001$ & 26903 & 538 & 2.00 & +35.0 & 0.03 & $0.48(0.44-0.52)$ \\
\hline \multicolumn{12}{|l|}{ UCD } \\
\hline IPF & NA & NA & NA & NA & NA & NA & 1863 & NA & & 0.92 & NA \\
\hline LC & NA & 2292943 & NA & -28.7 & $<0.001$ & NA & 8956 & NA & & 0.40 & $0.19(0.18-0.20)$ \\
\hline Pneumonia & NA & 1023 & NA & & 0.14 & NA & 19 & NA & & 1.00 & $3.83(2.43-6.04)$ \\
\hline \multicolumn{12}{|l|}{ Place of death } \\
\hline Inpatient & NA & 700652 & NA & -45.8 & $<0.001$ & NA & 5625 & NA & & 0.44 & $2.25(2.17-2.33)$ \\
\hline Home & NA & 993905 & NA & -22.5 & $<0.001$ & NA & 3687 & NA & & 0.11 & $0.66(0.63-0.68)$ \\
\hline Hospice & NA & 211291 & NA & +1130.2 & $<0.001$ & NA & 847 & NA & +8880.0 & $<0.001$ & $0.81(0.76-0.87)$ \\
\hline Nursing home & NA & 331624 & NA & -43.0 & $<0.001$ & NA & 896 & NA & & 0.55 & $0.52(0.49-0.56)$ \\
\hline
\end{tabular}

International Classification of Diseases (10th Revision) (ICD-10) codes used for underlying cause of death (UCD) were pulmonary fibrosis (J84.1 and J84.9), lung cancer (C34-C34.9) and pneumonia (J09-J18.9). Similar to prior studies, ICD-10 codes were used to identify and exclude patients with underlying connective tissue diseases (M32-M35, M35.1, M35.5, M35.8-M36 and M05-M08.9), radiation fibrosis (J70.1), sarcoidosis (D86-D86.9), pneumoconiosis (J60-J65) and hypersensitivity pneumonitis (J67-J67.9). If no data are shown for \% change in rate, the regression

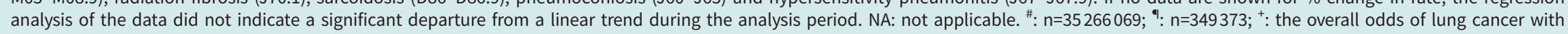
IPF compared to without IPF. 
This finding was observed irrespective of sex, age group, race or year of death. Our findings are consistent with a previous study by WeLls and MANnino [6] that reported LC in $4.8 \%$ of decedents with IPF compared to 6.5\% decedents in the general US population from 1979 to 1991.

Possible explanations for the lower odds of LC with IPF are a lower prevalence of LC in patients with IPF or improved survival in those with LC and IPF. Previous studies have shown that patients with both IPF and LC have a shorter survival time [7, 8]. This is likely due to surgical procedures and treatments for LC, which have both been associated with acute exacerbations of IPF [7]. As noted in this study, the mortality rates for LC patients without IPF improved over the study period, but in those with IPF and LC, a similar improvement was not noted. Therefore, the likely explanation for our findings is the lower prevalence of LC in patients with IPF. In contrast to our findings, two previous studies in European cohorts reported an increased incident rate of LC in patients with IPF compared to the general population [9, 10]. A systematic review evaluating comorbidities in IPF reported a wide range of LC prevalence rates ranging from $3 \%$ to $48 \%$ [11]. The majority of these studies were single-centred with small sample sizes, and therefore selection and referral bias could have influenced the results. Our study population was significantly larger than those in previous studies. In IPF compared to the general population, the higher LC incident rate but lower prevalence could also be explained by the shorter survival time associated with IPF. Ascertainment and surveillance biases may have contributed to the higher reported incidence since IPF patients are more likely to seek evaluation sooner due to respiratory symptoms and thus have chest computed tomography scans performed more frequently. Our non-IPF decedent group also probably represents a sicker population (similar to the IPF patients) and is, therefore, a more appropriate comparison in contrast to the prior studies where the control group was a live population. Presumably, the frequency of LC among decedents exceeds that in a live population.

In a recent study using the UK IPF registry, LC was present in only $0.6 \%$ of patients with IPF [12]. Similar to our estimates, in recent studies of IPF patients admitted to the intensive care unit, LC was reported in $2.3-3.5 \%$ of patients $[13,14]$. Since both LC and IPF have a short survival time, the lower prevalence reported in these studies and ours is likely to be because those with LC or IPF die before the other condition can develop. Unfortunately, we did not have data on the duration of the disease to determine whether long-term IPF patients have different rates of LC prevalence than short-term patients. However, given that our study extended over a 15-year period, we believe that our reported rates are reliable.

Consistent with other studies, we noted declining LC-related mortality rates in the non-IPF decedents [15]. However, in the IPF decedents, the overall rates were unchanged. The decreasing mortality rates in the 65-74-year age group is probably due to improved survival of patients with both IPF and LC in this age group. The increasing mortality rates in those $\geqslant 75$ years old are probably due to improved diagnosis or increased documentation of both diseases. The increase in LC rates in Hispanic people with IPF is probably due to improved access to medical care facilitated by the Affordable Care Act.

We noted that the odds of having LC listed as the UCD was even lower in IPF decedents compared to those without IPF. This is likely to be because a significant percentage of IPF patients experience an acute exacerbation and/or pneumonia prior to the death and are therefore likely to have those diagnoses listed as the UCD instead of LC. In the IPF and LC group, 20.8\% of decedents had IPF listed as the UCD. Although only a small percentage of decedents had pneumonia listed as the UCD in both groups $(0.24 \%$ of LC decedents without IPF and $1.16 \%$ of LC decedents with IPF), the odds ratio was higher in the IPF group (3.83, 95\% CI 2.43-6.04).

The strength of this study is the evaluation of the whole US population and the large numbers. The main drawback is that we are not able to verify the accuracy of the diagnosis or the documentation on the death certificate. Since the diagnosis of IPF may require multidisciplinary discussion and/or surgical lung biopsy, it is possible that under-reporting and misdiagnosis may have occurred. LC in early stages or remission could also be under-reported; however, this would probably not be affected by whether a person has IPF or not. Our findings are unlikely to be due to random chance alone given the large numbers. To support this claim, we analysed the prevalence of LC in decedents with COPD, a pulmonary condition that is associated with a higher prevalence of LC. The overall odds of having LC were higher in those with COPD (OR 1.59, 95\% CI 1.58-1.60) compared to those without COPD. In summary, we noted that the prevalence of LC is lower in decedents with IPF compared to those without IPF. 


\section{Niranjan Jeganathan $\oplus^{1}$, Derrick Cleland ${ }^{2}$ and Matheni Sathananthan ${ }^{2}$}

${ }^{1}$ Dept of Medicine, Division of Pulmonary, Critical Care, Hyperbaric, Allergy and Sleep Medicine, Loma Linda University Health, Loma Linda, CA, USA. ${ }^{2}$ Dept of Medicine, Loma Linda University Health, Loma Linda, CA, USA.

Corresponding author: Niranjan Jeganathan (njeganathan@llu.edu)

Provenance: Submitted article, peer reviewed.

Author contributions: N. Jeganathan and M. Sathananthan conceived the idea and designed the study. All authors acquired the data. N. Jeganathan analysed the data. The manuscript was drafted by all authors, and all authors contributed to the data interpretation and edited the manuscript.

Conflict of interest: None declared.

\section{References}

1 Raghu G, Collard HR, Egan JJ, et al. An official ATS/ERS/JRS/ALAT statement: idiopathic pulmonary fibrosis: evidence-based guidelines for diagnosis and management. Am J Respir Crit Care Med 2011; 183: 788-824.

2 Centers for Diseases Control and Prevention. Multiple Cause of Death 1999-2018. https://wonder.cdc.gov/ mcd.html. Date last accessed: 19 May 2021.

3 Dove EP, Olson AL, Glassberg MK. Trends in IPF-related mortality in the United States: 2000-2017. Am J Respir Crit Care Med 2019; 200: 929-931.

4 Jeganathan N, Smith RA, Sathananthan M. Mortality trends of idiopathic pulmonary fibrosis in the United States from 2004 through 2017. Chest 2021; 159: 228-238.

5 Jeganathan N, Miot C, Sathananthan A, et al. The association of pulmonary fibrosis with diabetes mellitus. ERJ Open Res 2020; 6: 00237-2020.

6 Wells C, Mannino DM. Pulmonary fibrosis and lung cancer in the United States: analysis of the multiple cause of death mortality data, 1979 through 1991. South Med J 1996; 89: 505-510.

7 Brown SW, Dobelle M, Padilla M, et al. Idiopathic pulmonary fibrosis and lung cancer. A systematic review and meta-analysis. Ann Am Thorac Soc 2019; 16: 1041-1051.

8 Tomassetti S, Gurioli C, Ryu JH, et al. The impact of lung cancer on survival of idiopathic pulmonary fibrosis. Chest 2015; 147: 157-164.

9 Hubbard R, Venn A, Lewis S, et al. Lung cancer and cryptogenic fibrosing alveolitis. A population-based cohort study. Am J Respir Crit Care Med 2000; 161: 5-8.

10 Le Jeune I, Gribbin J, West J, et al. The incidence of cancer in patients with idiopathic pulmonary fibrosis and sarcoidosis in the UK. Respir Med 2007; 101: 2534-2540.

11 Raghu G, Amatto VC, Behr J, et al. Comorbidities in idiopathic pulmonary fibrosis patients: a systematic literature review. Eur Respir J 2015; 46: 1113-1130.

12 Spencer LG, Loughenbury M, Chaudhuri N, et al. Idiopathic pulmonary fibrosis in the UK: analysis of the British Thoracic Society electronic registry between 2013 and 2019. ERJ Open Res 2021; 7: 00187-2020.

13 Mooney JJ, Raimundo K, Chang E, et al. Mechanical ventilation in idiopathic pulmonary fibrosis: a nationwide analysis of ventilator use, outcomes, and resource burden. BMC Pulm Med 2017; 17: 84.

14 Schrader M, Sathananthan M, Jeganathan N. Patients with idiopathic pulmonary fibrosis admitted to the ICU with acute respiratory failure - a reevaluation of the risk factors and outcomes. J Intens Care Med 2021; in press [https://doi.org/10.1177/0885066621989244].

15 Howlader N, Forjaz G, Mooradian MJ, et al. The effect of advances in lung-cancer treatment on population mortality. N Engl J Med 2020; 383: 640-649. 